\title{
Aplicação do Sistema Multidimensional de Categorização de Comportamentos em sessões de coaching
}

\section{Application of Multidimensional System for coding Behavior in coaching sessions \\ Aplicación del Sistema Multidimensional de Categorización de los Comportamientos en sesiones de coaching}

\author{
Nicodemos Borges ${ }^{1}$, Camila Quintella Gonçalves $^{2}$, Rute Brasil Pinto ${ }^{3}$
}

\begin{abstract}
[1] Instituto Nicodemos Borges e Universidade São Judas [2] [3] Universidade São Judas I Título abreviado: Categorização de comportamentos em sessões de coaching | Endereço para correspondência: Rua Itapeva, 26 - Conj. 901 - Bela Vista - São Paulo - SP - CEP: 01332-000 | Email: nicodemos@nicodemosborges. com.br I DOI: 10.18761/pac.2016.029
\end{abstract}

\begin{abstract}
Resumo: O coaching é um processo de intervenção voltado para o desenvolvimento de repertório cuja demanda tem aumentado nos últimos anos. Apesar deste aumento, são poucas as pesquisas que investigam o coaching, sendo ainda mais raras investigações focadas em estudos de processo. Dado esse cenário, lançou-se mão de um método utilizado para investigar processos clínicos para investigar um processo de coaching, pois, assim como a psicoterapia, o coaching também é uma modalidade de intervenção que ocorre em um sistema de parceria profissional-cliente. A presente pesquisa investigou comportamentos verbais vocais que ocorreram na interação entre coach e coachee num processo de coaching. Para isso, utilizou-se o Sistema Multidimensional para a Categorização de Comportamentos na Interação Terapêutica. Os resultados obtidos indicam que os tipos de comportamentos do coach mais comuns são Informação, Solicitação de relatos e Solicitação de reflexões; enquanto que os do cliente são Relato e Estabelecimento de relação. Além disso, quando comparados a resultados de pesquisas a respeito de psicoterapia, parece haver diferença, sendo que nesta última modalidade de intervenção parece haver maior incidência de comportamentos de Interpretação e Empatia que em processos de coaching. Os resultados sugerem também que processos de coaching parecem direcionados para que o coachee adquira auto-observação, autoanálise e autocontrole.
\end{abstract}

Palavras-chave: Processo de coaching, Análise do Comportamento, Psicologia do Coaching, Sistema de categorização de comportamentos. 
Abstract: Coaching is an intervention process oriented for repertoire development. The demand for coaching has increased in the past few years. Despite this increase, there are few researches investigating coaching, and investigations focused on procedure studies are even rarer. As coaching, like psychotherapy, is also a type of intervention that occurs in a professional-client partnership system, in the present study a method used to investigate clinical processes was applied to the investigation of coaching. This research investigated the verbal behaviors that occur in the interaction between coach and coachee in a coaching process. Thereby, the Multidimensional System for Coding Behavior in Therapeutic Interaction was implemented. The results point out that coaches' most common behaviors are Information, Requesting of reports, Requesting of reflection, whereas coachees' most common behaviors are Reporting and Establishing relations. In addition, when compared to results of psychotherapy-oriented researches, there appears to be some difference, for in the latest behaviors of Interpretation and Empathy seems to occur more often than in coaching processes. The results also suggest that coaching processes seem aimed at the coachee acquiring self-observation, self-analysis, and self-control.

Keywords: Coaching process, Behavior Analysis, Coaching Psychology, Coding behavior system.

Resumen: El coaching es un proceso de intervención direccionado al desarrollo de repertorio cuya demanda ha aumentado en los últimos años. A pesar de este aumento, son pocos los estudios que investigan el coaching, y aún más raras las investigaciones centradas en estudios de proceso. Ante esta dificultad, se ha manejado a la investigación de un proceso de coaching un método empleado para investigar los procesos clínicos, pues, tal como el coaching, la psicoterapia es también una modalidad de intervención que ocurre en un sistema de asociación profesional-cliente. Esta investigación ha estudiado conductas verbales que se han producido en la interacción entre coach y coachee en el proceso de coaching. Para esto, se utilizó el Sistema Multidimensional para la Categorización de Conductas en la Interacción Terapéutica. Los resultados indican que los tipos de conductas más comunes del coach son Información, Solicitud de relatos, Solicitud de reflexiones, en cuanto los del coachee son Relato y Estabelecimiento de relación. Además, cuando comparados con resultados de investigaciones relativas a psicoterapia, parece haber diferencia, pues que en esta última modalidad de intervención parece haber más incidencia de conductas de Interpretación y Empatía que en procesos de coaching. Los resultados también indican que procesos de coaching parecen direccionados para que el coachee adquiera auto-observación, autoanálisis y autocontrol.

Palabras-clave: Proceso de Coaching, Análisis de la Conducta, Psícologia del coaching, Sistema de Categorización de Conductas. 
A globalização econômica, política e tecnológica são fatores que influenciam diretamente no desenvolvimento social. $\mathrm{O}$ avanço destes fatores fez com que aumentassem as exigências para os indivíduos se tornarem cada vez mais flexíveis, ágeis e se adaptarem às mudanças, alcançando assim seu autogerenciamento e desenvolvimento pessoal e profissional (Elisa \& Yoshida, 2007). Ainda de acordo com Elisa e Yoshida (2007), para os indivíduos se adaptarem a todas as exigências feitas pela sociedade, seja no âmbito profissional ou pessoal, se faz necessário a busca por profissionais capacitados que os direcionem para atingirem seu autodesenvolvimento.

Um destes profissionais, que há muito tempo é procurado, é o terapeuta. Segundo Meyer e Vermes (2001), o trabalho exercido pelo terapeuta tem como função promover aumento das contingências reforçadoras da vida do cliente através de mudanças comportamentais que façam com que ocorra a minimização do sofrimento do indivíduo. As autoras ainda ressaltam que o terapeuta é um profissional capacitado para utilizar procedimentos específicos que venham a auxiliar o cliente.

Starling (2010) aponta que "é tarefa do terapeuta explorar, em benefício do cliente, as diversas técnicas disponíveis para o manejo do seu problema e auxiliá-lo a mudar o seu comportamento na direção por ele desejada." (p. 226). Ireno (2007) diz que as habilidades e conhecimentos adquiridos pelo terapeuta, poderão ajudar o cliente que o procura a resolver seus problemas.

Oliveira e Borges (2007) descrevem que o terapeuta é procurado para ajudar o cliente mudar a forma como pensa e age em determinadas situações e, também, auxiliar o cliente a responder de forma que poderá gerar consequências que diminuam o sofrimento vivenciado em determinada situação.

Assim, entende-se que o terapeuta é o profissional buscado com intuito de prestar auxílio ao cliente, cujas técnicas e conhecimentos utilizados por este profissional deverão ter como objetivo ajudar o cliente a solucionar a demanda apresentada na terapia.

A respeito da psicoterapia, alguns autores apresentam diferentes definições, dentre eles Guilhardi (2004) aponta que a terapia se define por interações entre cliente e terapeuta e tem o objetivo de alterar comportamentos do cliente a partir de técnicas comportamentais, com foco nas demandas trazidas pelo cliente ou familiares. Moura e Venturelli (2004) definem terapia como um processo direcionado pelo terapeuta, com sequência lógica e organizada de procedimentos psicológicos que produzem mudanças comportamentais no cliente.

De acordo com Marçal (2010), o termo Terapia Comportamental foi introduzido por Skinner em 1953 num projeto de pesquisa, mas só foi utilizado em uma produção científica em 1954. Contudo, se considerarmos que as práticas de intervenção sobre comportamentos respondentes também devem ser tidas como parte desse tipo de psicoterapia, pode-se dizer que a Terapia Comportamental inicia a partir de 1920 (Sant'Ana, 2004), com John B. Watson.

As décadas de 70 e 80 foram marcadas pelo surgimento da terapia cognitivo-comportamental, já a década 90 foi quando emergiu a análise do comportamento clínica (Simões Filho, 2014), que seria um dos nomes mais atualizados para se referir a Terapia Comportamental. De acordo Pérez-Alvarez (2006, citado por Simões Filho, 2014), a ascensão da terapia comportamental ocorreu devido ao "desenvolvimento da análise do comportamento, do behaviorismo radical, como um desdobramento da análise funcional, sobretudo no campo do comportamento verbal, por conta de limitações da terapia cognitivo-comportamental" (p. 4).

Para Vandenbergue (2011), a terapia comportamental no Brasil é nomeada como terapia analítico-comportamental e se desenvolveu a partir da análise do comportamento. $\mathrm{O}$ autor ainda aponta que outros nomes têm sido utilizados para se referir a essa prática, tais como: Análise Comportamental Clínica e Terapia por Contingências de Reforçamento. Além disso, pode-se considerar que as práticas de Terapia Comportamental Dialética (DBT), Psicoterapia Analítica Funcional - FAP e a Terapia de Aceitação e Compromisso - ACT, tratam-se de modelos terapêuticos que integram a Terapia Comportamental ou Analítico-Comportamental.

A despeito da falta de consenso sobre seu nome ou da data de seu início, a Terapia Comportamental tem acumulado conhecimento prático e científico a respeito de sua prática (Banaco, 2012), facilitando inclusive na formação de novos profissionais para atuarem nessa área. 
Além do terapeuta, outro profissional buscado atualmente para ajudar os clientes a se desenvolverem é o coach. Silva (2010) cita o coach como o profissional responsável pela condução do processo de coaching, aquele que irá auxiliar o coachee (cliente de coaching) a alcançar seus objetivos de autodesenvolvimento. A autora levanta a importância do coach ter amplo conhecimento e formação teórica em temas relacionados ao desenvolvimento humano, além de uma vasta experiência profissional.

Para Bloch, Mendes e Visconte (2012), o coach deve liderar o coachee no processo de busca pelo alcance do autodesenvolvimento, para isso o coach deve levar o indivíduo a fazer questionamentos para que atinja o seu objetivo; sendo que, segundo Marques (2013), é por meio de questionamentos que o coach induz o coachee às reflexões. Além disso, o último autor também destaca que o papel do profissional de coaching é apoiar o coachee, acompanhando-o e mensurando os resultados de suas ações, bem como motivá-lo até alcançar suas metas.

A respeito do coaching, Franciscato, Ferreira e Walter (2010) o definem como um processo que contribui na obtenção de resultados mais rápidos e com qualidade, e que auxilia no descobrimento de novos talentos. Ascama (2004) define o coaching como a relação entre coachee e coach, sendo este último o responsável por utilizar diversas técnicas e métodos com a finalidade de ajudar o cliente identificar e atingir seus objetivos.

Borges (2015) se refere ao coaching como uma modalidade de intervenção que visa promover repertório de autogerenciamento norteado por objetivos de desenvolvimento. Para isso, o profissional de coaching lança mão de técnicas e procedimentos de intervenção, sendo o trabalho norteado por um objetivo comportamental ou produto de comportamento, o qual deve ser delineado no início do processo de coaching pelo cliente, com o auxílio do profissional.

Parte da comunidade de coaching aponta que o processo surgiu em meados dos anos de 1970 no meio esportivo, em específico no Tênis (Oliveira, 2009). Já para Chiavenato (2002), o coaching não é um processo novo, sendo este criado e inspirado pelo filósofo Sócrates. Segundo o autor, este processo que atualmente é intitulado como coaching, era utilizado pelo filósofo como método pedagógico de ensinar as pessoas a pensar e a refletir para buscar o conhecimento "no fundo de si mesmas". O autor ressalta que, após aproximadamente 2.500 anos, a forma de trabalho desenvolvida antigamente por Sócrates é a inspiração para o coaching. Assim, é possível afirmar que, assim como no caso da terapia comportamental, não há consenso a respeito da origem do coaching.

A respeito das duas modalidades de intervenção citadas, terapia comportamental e coaching, pode-se observar que os diversos autores afirmam tratarem-se de processos que ajudam na melhora do repertório comportamental do indivíduo. A esse respeito, Franchi e Sierra (2007) afirmam que o trabalho do coach, assim como o do terapeuta, pode ser entendido como processo que auxilia o cliente no seu autodesenvolvimento e no crescimento profissional e pessoal.

Borges (2015) reafirma que tanto o coaching quanto a terapia comportamental são modalidades de intervenções cujos objetivos são auxiliar as pessoas em seu desenvolvimento, sendo ambas intervenções autocorretivas buscadas pelo próprio cliente.

Ainda de acordo com Borges (2015), apesar de haver semelhanças entre essas práticas, também há diferenças. Entre as distinções, uma das principais parece estar relacionada ao tipo de demanda que leva o cliente a procurar uma ou outra modalidade de intervenção. No caso da terapia, os clientes geralmente estão em sofrimento e buscam ajuda para mudar as contingências que diminuam as condições aversivas. Já no coaching, os clientes procuram o coach sem sofrimento aparente, a ajuda seria por manejar contingências de modo a produzir mais condições apetitivas. O autor considera que essas diferentes contingências é que controlam a afirmação de muitos autores que afirmam que terapia é para população clínica enquanto que coaching é para população não-clínica. Além disso, é mais consensual na área de coaching que pessoas em condição de vulnerabilidade não estão aptas a processos de coaching, não estão coachable.

Apesar de haver muita discussão a respeito de coaching, seu corpo de conhecimento científico ainda carece de desenvolvimento. Blanco (2006) afirma que é escassa a produção acadêmica sobre coaching, sendo ela encontrada, principalmente em 
bases internacionais das áreas de Administração e Psicologia. Borges (2015) corrobora Blanco e afirma que a terapia é uma modalidade de intervenção cujo corpo de conhecimento científico está bem mais estabelecido que a do coaching. Esta última, por ser uma modalidade de intervenção relativamente nova, ainda apresenta poucos estudos que avaliam seus resultados. Apesar de poucos, já há evidências consideráveis que essa modalidade de intervenção promove resultados (Grant, 2013).

Apesar de haver estudos que indicam a efetividade dessa modalidade de intervenção, Borges (2015) afirma que há carência de estudos que demonstrem o que ocorre nesse processo, sendo esses necessários pois poderão lançar luz sobre variáveis responsáveis pelos resultados obtidos dentro desses pacotes de intervenção, como já se tem na área da psicoterapia.

Diante do exposto julga-se necessários estudos a respeito de coaching, especialmente estudos de processo, visando mapear o que ocorre nessa modalidade de intervenção que pode ser responsável pelos seus resultados. Tal necessidade torna-se ainda mais evidente ao se considerar o prognóstico de Karawejczyk, e Cardoso (2012), que a demanda pelo coaching está crescendo a cada ano e possui perspectiva de continuar expandindo.

$\mathrm{Na}$ área de investigação de processos clínicos, é possível encontrar diversos autores que vêm se debruçando sobre o tema (e.g., Banaco, 1993; Braga \& Vandenberghe, 2006; Del Prette, Del Prette, \& Meyer, 2007; Guedes, 1993). Banaco, em 1993, já descrevia que inúmeros trabalhos estavam sendo realizados a respeito das características e habilidades pessoais do terapeuta que poderiam contribuir para os resultados da intervenção.

Por não se dispor de instrumentos consagrados para estudo de processo em coaching e por esses já estarem mais avançados na terapia, o presente trabalho lançará mão de um instrumento desenvolvido para estudar interação terapeuta-cliente para investigar a interação coach-coachee. Mais especificamente, será utilizado o Sistema Multidimensional para a Categorização de Comportamentos na Interação Terapêutica (SiMCCIT), desenvolvido por Zamignani (2007).

De acordo com Zamignani e Meyer (2011), o SiMCCIT foi desenvolvido para estudar a intera- ção terapêutica, descrevendo os comportamentos envolvidos nesta relação. Após a realização de uma avaliação sistemática da literatura sobre categorização de eventos verbais da interação terapêutica, Zamignani (2007) afirma não ter encontrado um sistema considerado suficiente que possibilitasse a formulação de um instrumento de categorização e estudo da psicoterapia analítico-comportamental, o que o levou a desenvolver o SiMCCIT.

Moskorz e Kubo (2012) ressaltam que as informações obtidas na tese de Zamignani (2007) podem auxiliar o terapeuta a compreender comportamentos da interação entre terapeuta e cliente que possibilite possíveis modificações comportamentais. Os autores apontam que, além disso, o SiMCCIT pode ajudar terapeutas recém-formados a entenderem comportamentos constituintes da interação terapêutica.

Segundo Zamignani e Meyer (2014), desde que o SiMCCIT foi criado em 2007, muitos pesquisadores vêm o utilizando em diversos estudos, realizando a comparação entre dados, contribuindo para a validação e aperfeiçoamento do sistema (e.g. Oliveira, 2014; Moskorz e Kubo, 2012; Peron, 2012; Silveira, 2009; Simões Filho, 2014; Vernucio, 2012).

Oliveira (2014) desenvolveu um estudo cujo objetivo foi analisar sessões de terapia comportamental com base na aplicação das categorias do SiMCCIT. A autora dividiu o trabalho em dois estudos, o Estudo 1 dedicado a analisar os comportamentos do terapeuta e o Estudo 2 os comportamentos do cliente.

O Estudo 1 foi feito com a participação de três terapeutas, psicólogos e analistas do comportamento com clientes que estavam em processo terapêutico há no mínimo três meses. Os três terapeutas gravaram uma dessas sessões, com a permissão dos clientes, e posteriormente as concederam à pesquisadora. De posse das gravações, a pesquisadora fez a transcrição das três sessões e aplicou o SiMCCIT. Para realização do Estudo 2, foram utilizadas as mesmas sessões, porém empregaram-se as categorias, do mesmo sistema, referentes aos comportamentos dos clientes.

De acordo com Oliveira (2014) ambos estudos apontam que o terapeuta modela o comportamento do cliente ao longo do processo terapêutico. Os resultados destes estudos também remetem que te- 
rapeutas de uma mesma formação parecem iniciar a sessão e conduzi-la de forma que facilite o relato do cliente. Para isso, solicitam relatos, reflexões, são empáticos e interpretam. Ainda é visto que clientes de semelhante faixa etária parecem comportar-se de maneira similar, mesmo com queixas diferentes. No decorrer da terapia, estabelecem relações entre fatos, fazem relatos, traçam metas, concordam e pouco se opõem ao que é dito pelo profissional.

Nesta mesma linha, Zamignani e Meyer (2014) desenvolveram outro estudo com base no SiMCCIT. Tal pesquisa teve como objetivo testar a aplicação do SiMCCIT na categorização de três sessões terapêuticas (sessões 3, 11 e 17), para representar três diferentes momentos do processo terapêutico. $\mathrm{O}$ estudo contou com a participação de um terapeuta analítico-comportamental, com 25 anos de experiência clínica e uma cliente de 32 anos, com queixas relacionadas a problemas matrimoniais. As sessões foram registradas pelo pesquisador, com a autorização dos participantes, por meio de gravação de vídeo e para realizar a categorização dos comportamentos foi utilizado o software The Observer.

Os autores realizaram uma análise descritiva das sessões, visando identificar padrões de interação que ocorreram durante as três sessões selecionadas. Dentre os resultados obtidos, Zamignani e Meyer (2014) apresentaram a duração e o total de ocorrências das categorias de respostas verbais do terapeuta e do cliente nas sessões analisadas.

Zamignani e Meyer (2014) discorrem que é comum que no início da terapia o profissional solicite mais relatos (de informação) devido ao profissional possuir poucos dados a respeito do cliente. Contudo, no caso que analisaram isso não aconteceu, pois desde as primeiras sessões houve verbalizações espontâneas por parte da cliente. Assim, as categorias de comportamentos do terapeuta mais frequentes foram Interpretação, Solicitação de reflexões e Aprovação. Enquanto as categorias mais frequentes do cliente foram Relatos (de informações), Estabelecimentos de relações e Concordâncias (com o que era dito pelo terapeuta).

Sadi e Meyer (2014) também realizaram um estudo com base no SiMCCIT, neste caso foi categorizado um processo terapêutico de um cliente diagnosticado com Transtorno de Personalidade Borderline. O objetivo da pesquisa foi de relacionar a interação da terapia com o abandono do tratamento. Esse estudo contou com a participação de uma terapeuta analítico-comportamental, que assumiu a posição também de pesquisadora; e uma cliente, que já havia abandonado processos terapêuticos anteriores. O processo terapêutico cujos dados foram utilizados contava com 22 sessões, sendo que deste total apenas quatorze foram gravadas e transcritas. Após a $22^{\circ}$ sessão, a cliente abandonou o processo terapêutico e as autoras fizeram uma análise das sessões a partir da categorização das sessões transcritas, a fim de compreenderem melhor a interação terapêutica que havia sido estabelecida.

Os resultados da pesquisa de Sadi e Meyer (2014) indicaram que houve uma diminuição nas categorias Empatia e Facilitação. Apesar destas categorias mostrarem-se importantes para o estabelecimento e manutenção do vínculo, auxiliarem na criação de um ambiente terapêutico não punitivo e acolhedor com um cliente borderline, as autoras ressaltaram que houve uma diminuição na ocorrência destas categorias. Sadi e Meyer (2014) justificaram que este resultado pode estar relacionado ao crescimento da frequência de relatos da cliente, sem estabelecer relações, com a possível intenção de esquivar-se de temas aversivos ou que remetessem algum vínculo entre terapeuta-cliente.

É possível verificar que o uso de categorização da interação entre o profissional e o cliente nas pesquisas de clínica analítico-comportamental tem ajudado a lançar luz sobre os aspectos relevantes desta interação, o que não só permite o aperfeiçoamento daquela prática como também pode contribuir com a formação de novos profissionais. Diante disso, O presente estudo pretende utilizar o SiMCCIT (Zamignani, 2007) para investigar as interações envolvidas entre o profissional e o cliente em processos de coaching. Mais especificamente, este estudo teve como objetivo categorizar os comportamentos verbais vocais do profissional e do cliente que ocorrem em um processo de coaching. 


\section{Método}

\section{Participantes}

Profissional e cliente de coaching:

a) O profissional foi um psicólogo de 37 anos, com formação em coaching, especialista em terapia comportamental, mestre e doutor em análise do comportamento.

b) O cliente foi um psicólogo de 45 anos que atua como clínico. A demanda para buscar o serviço de coaching foi desenvolver "autonomia de pensamentos", o que foi definido por Borges (2015) como comportamento que se caracteriza por tomadas de decisão sob controle de seus valores pessoais e demais contingências envolvidas nas situações vivenciadas e que se contrapõe a comportamentos dependentes, em que as decisões são tomadas sob forte influência de orientações de pessoas de seu meio.

Os participantes assinaram o Termo de Consentimento Livre e Esclarecido (TCLE) autorizando a gravação das reuniões e utilização dos dados para pesquisas.

\section{Material/Instrumento}

Os materiais utilizados foram:

a)Vídeo de duas sessões de coaching para treino sistemático de categorização ("piloto").

b) Transcrição das duas sessões "piloto".

c) Vídeo de três sessões de coaching.

d) Transcrição das três sessões referidas no item anterior.

e) Software de Treino de Observadores do SiMCCIT desenvolvido por Zamignani e Meyer - 2007.

f) Manual do SiMCCIT desenvolvido por Zamignani e Meyer - 2007

g) Sistema Multidimensional para a caracterização de comportamentos na interação terapêutica (SiMCCIT).

O SiMCCIT é composto por três eixos de categorização, uma vez que cada um deles representa uma dimensão ou aspecto do comportamento dos participantes. O Eixo I diz respeito ao Comportamento
Verbal Vocal e tem como qualificador o tom emocional e gestos ilustrativos; o Eixo II analisa os temas abordados; o Eixo III corresponde às Respostas Motoras.

No presente estudo foi utilizado apenas o Eixo I. Este é desenvolvido para categorizar os comportamentos verbais vocais do profissional e do cliente. Ele contém 10 categorias para a verbalização do profissional e oito para a verbalização do cliente. As categorias do profissional são: Solicitação de relato, Facilitação, Empatia, Informação, Solicitação de reflexão, Recomendação, Interpretação, Aprovação, Reprovação e Outras vocal profissional ${ }^{1}$; e, as categorias do cliente são: Solicitação, Relato, Melhora, Metas, Relações, Concordância, Oposição e Outras vocal cliente.

\section{Procedimento}

Dois dos pesquisadores passaram por um treino sistemático para observadores do SiMCCIT, primeiro utilizando-se do software de Treino sistemático de observadores para as categorias do terapeuta e do cliente, desenvolvido por Zamignani (2007) e cedido pelo autor. Posteriormente, os comportamentos de categorizar foram treinados por meio da classificação de duas sessões de Coaching. Apenas quando o nível de concordância ultrapassou 95\% iniciou-se a análise dos resultados utilizados nesta pesquisa.

\section{a) Seleção dos dados para análise}

A ideia foi categorizar três sessões, sendo uma mais inicial, uma intermediária e uma mais próxima ao final do processo. As sessões 1 e 10 foram descartadas por serem muito específicas, a primeira consistindo de avaliações, fechamento de objetivos a serem alcançados até o final do processo e explicações a respeito do coaching; a última por se caracte-

1 O Sistema Multidimensional para a Categorização de Comportamento na Interação Terapêutica foi originalmente feito para estudar interações da díade terapeuta-cliente, mas neste artigo foi utilizado para categorizar os comportamentos verbais do coach - coachee, sendo o único ajuste, a alteração do nome da categoria "OUTRAS VOCAL TERAPEUTA" para "OUTRAS VOCAL PROFISSIONAL", tornando-se assim adequada para categorizar qualquer díade em que há um profissional e um cliente, como são os casos da psicoterapia e do coaching. 
rizar por avaliações de desempenho do cliente, do profissional e do processo, além de encerramento.

Desta forma, foram selecionadas as sessões 2, 5 e 9. A duração de cada uma dessas sessões foi distinta, como é característico do processo de coaching. Assim, a Sessão 2, teve duração de $01 \mathrm{~h} 57 \mathrm{~m} 14 \mathrm{~s}$, a Sessão 5 durou $01 \mathrm{~h} 45 \mathrm{~m} 16 \mathrm{~s}$ e a Sessão 9 durou 01h11m58s.

\section{b) Preparação dos dados para caracterização}

A partir das gravações foi realizada a transcrição na íntegra e individualmente das três sessões.

\section{c) Procedimento para a caracterização}

Cada sessão foi observada, transcrita e categorizada com base no sistema multidimensional para categorização de comportamentos da interação terapêutica (Zamignani, 2007), obtendo-se as frequências dos comportamentos vocais do terapeuta e do cliente individualmente em cada categoria e subcategoria do Eixo I, já mencionados. Posteriormente foi calculado o percentual de ocorrência de cada categoria de comportamento do profissional e do cliente e a quantidade de ocorrência de suas verbalizações.

\section{Resultados e discussão}

Este estudo se propôs analisar a categorização dos comportamentos verbais vocais do cliente e do profissional de coaching que ocorreram em três encontros (02, 05 e 09), utilizando-se do SiMCCIT.

Os resultados apresentados a seguir, foram comparados com outros estudos realizados com base no Sistema Multidimensional e discutidos comparativamente a partir de estudos de processos terapêuticos que utilizaram o mesmo sistema de categorização, uma vez que não foram encontrados estudos de coaching que façam categorizações dos comportamentos dos participantes durante o processo.

De acordo com Del Prette e Meyer (2014), a busca e comparação de estudos que utilizaram o SiMCCIT pode trazer mais conhecimento referente a processos de intervenção, uma vez que esta comparação traz a possibilidade de consulta e confrontação em novas pesquisas. Assim, busca-se que estes benefícios também ocorram na análise dos resultados obtidos na categorização de processos de coaching.
Na Figura 1 é apresentada a distribuição percentual e comparativa das categorias de verbalizações do coach, presentes no Eixo I.

Observando os resultados decorrentes da análise das sessões de coaching, verifica-se que as categorias mais frequentes no processo de coaching analisado foram Informação, Solicitação de relato, Solicitação de reflexão e Aprovação. Enquanto outras foram pouco frequentes, como Reprovação, Empatia, Interpretação e Recomendação.

Tais resultados estão em linha com que Bloch, Mendes e Visconte (2012) afirmam que deve ocorrer num processo de coaching. Segundo os autores, o papel do profissional é levar o cliente a buscar alternativas por meio de aplicação de ferramentas que geram autoconhecimento ao coachee; não cabendo ao profissional dar as direções (Recomendação) e nem analisar o comportamento do cliente (Interpretação). Bloch, Mendes e Visconte (2012) ainda afirmam que o coaching é um processo estruturado em que o coach ajuda o coachee a refletir (Solicitação de reflexão) para que ele tenha ações e se engaje para alcançar seu autodesenvolvimento.

Como pode-se verificar nos resultados deste estudo, uma das categorias que aparenta ser frequente no coaching, é Solicitação de reflexão. Em pesquisas cujo foco foi a díade terapeuta-cliente, se observa que esta mesma categoria também foi frequente (e.g. Oliveira, 2014; Zamignani \& Meyer, 2014). De acordo com Zamignani e Meyer (2014) Solicitação de reflexão ajuda o cliente estabelecer relações entre eventos, auxiliando-os a analisar funcionalmente seu comportamento. Para Karawejczyk e Cardoso (2012) o coach deve dirigir o processo de coaching de forma que este venha estimular o coachee a pensar em respostas para suas dificuldades e incentivá-lo a refletir para encontrar possibilidades de resolver problemas. Assim, esse tipo de comportamento verbal vocal parece comum por parte dos profissionais em ambas modalidades de intervenção, terapia e coaching.

Ainda em relação à categoria Solicitação de reflexão, verifica-se que houve uma diminuição de ocorrência de respostas dessa categoria ao longo das reuniões. Tal dado pode se dar pelo cliente estar desenvolvendo o repertório de autogerenciamento, que é uma característica do processo de coaching. Segundo Borges (2015) um dos principais repertórios desenvolvidos num processo de coaching é 


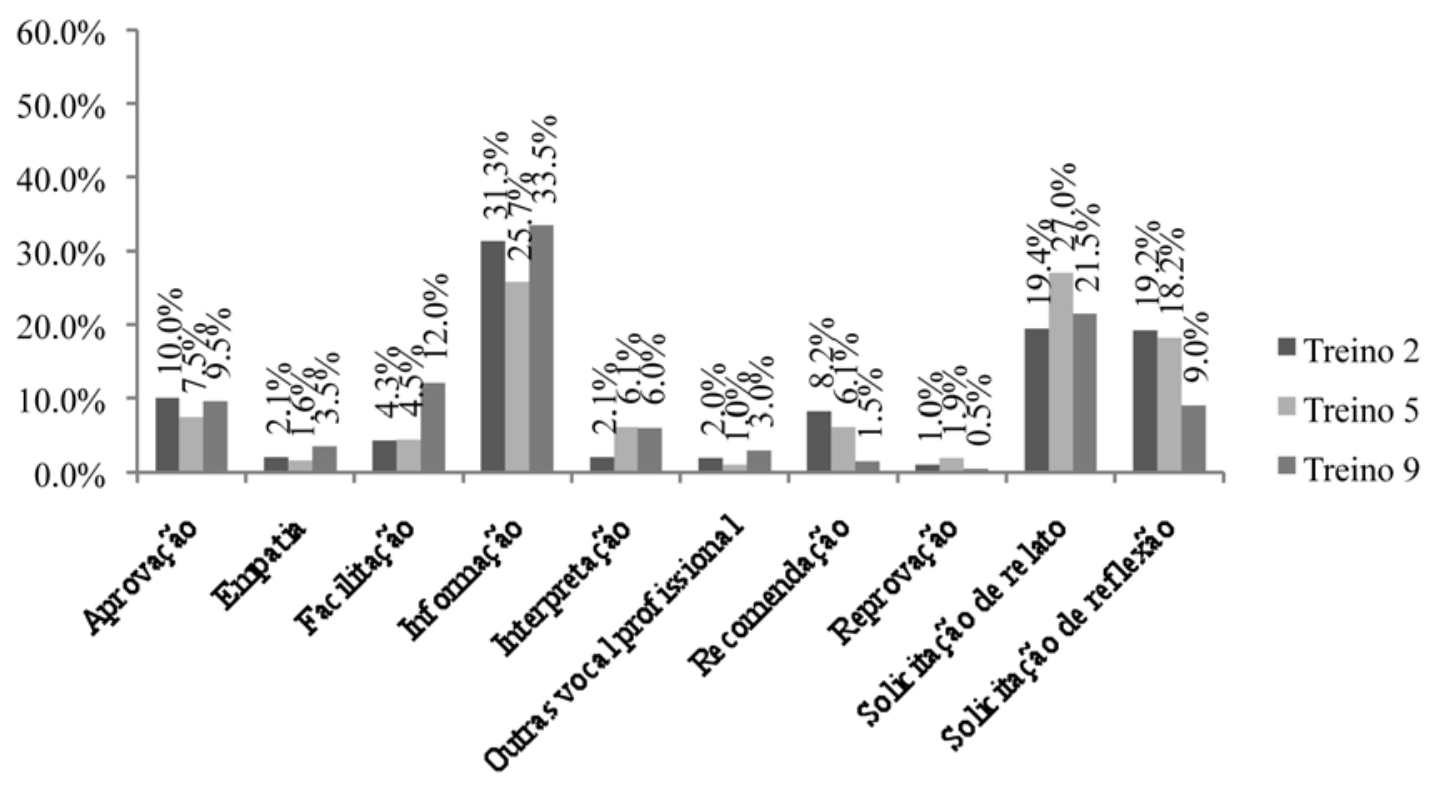

Figura 1: Percentual de ocorrência dos comportamentos verbais vocais emitidos pelo coach durante as sessões $n^{\circ} 02,05$ e 09.

autogerenciamento, entendido como capacidade do cliente de observar e interpretar as contingências das quais seu comportamento é função.

Outro ponto em comum observado no coaching e na terapia foi o baixo índice da categoria Reprovação, que parece ser pouco frequente em ambos os processos. Tal resultado indica que não aparenta ser comum o coach e nem o terapeuta discordarem, criticarem e se oporem ao que o cliente diz. De acordo com Vergara, Oliveira e Ferreira (2010) o coach deverá estabelecer um vínculo com o coachee, para isso deverá apoiá-lo, conduzi-lo e não impor o que deverá ser feito pelo cliente. $\mathrm{O}$ coach deve ser um facilitador do processo, não o seu condutor. Tal característica também é defendida por alguns terapeutas na condução do processo psicoterápico, como por exemplo Oliveira (2014), que defende que o terapeuta deve agir como um "facilitador investigativo e como uma audiência não punitiva” (p. 238).

Uma categoria que se mostrou frequente no processo de coaching foi Informação. Essa parece ser uma característica que distingue o coaching da terapia, pois de acordo com alguns estudos a respeito do processo terapêutico (e.g. Oliveira, 2014; Zamignani \& Meyer, 2014) essa categoria é uma das que apresenta menor ocorrência de respostas por parte do clínico.
A alta frequência de ocorrências de Informação no coaching pode ser compreendida por ser este um processo em que o coach apresenta recursos (ferramentas) para o coachee e por esse utilizar de comunicação direta - observações que faz a respeito do comportamento do coachee e que podem auxiliá-lo em seu processo de autoconhecimento.

Outro ponto observado que parece distinguir as duas práticas, é que a categoria Interpretação apresentou-se com menor frequência no coaching que na terapia, considerando os estudos de Oliveira (2014) e Zamignani e Meyer (2014). Tal resultado deve ter relação com a proposta do coaching, em que o coach deve ter o papel de parceiro, de apoiador do cliente, para que este por si só encontre os caminhos para atingir as metas e os resultados traçados (Campos \& Pinto, 2012). Para Borges (2015) o coach ensina o cliente a auto-observar, autoanalisar e autocontrolar seus comportamentos. Não cabe em processo de coaching interpretar, no máximo dar devolutivas de avaliações padronizadas. Já em processos psicoterápicos, o profissional lança mão de interpretações, que de acordo com Zamignani e Meyer (2014), são apresentações de explicações entre eventos e ocorrência de comportamentos do cliente, diagnósticos, devolutivas, etc. 
Uma das categorias que se apresentou com baixa frequência no processo de coaching analisado foi Empatia. Os resultados apontam que aparentemente esta categoria não pareceu ser a principal característica do papel do coach. Talvez porque não é característica do coaching ser acolhedor (no sentido de cuidar, "dar colo") com o cliente. Apesar da pouca frequência a Empatia ocorreu em todas as reuniões categorizadas, possivelmente pois apesar do coaching se tratar de uma intervenção objetiva e diretiva, é importante que o coach aceite, e seja apoiador do coachee, contribuindo para a busca por melhora de desempenho (Clutterbuck, 2008).

Assim, entende-se que apesar da baixa ocorrência de Empatia, ela se faz presente na prática do coaching, mas não como principal categoria inerente ao papel do coach. Nas pesquisas sobre processo psicoterápico, foi possível encontrar resultados que sugerem frequência semelhante às encontradas neste estudo (e.g. Oliveira, 2014; Zamignani \& Meyer, 2014).

Na Figura 1 também foi possível observar que todas as categorias ocorreram em todos os encontros, e comparando cada uma delas entre as três sessões, observa-se que a categoria Informação ocorreu mais frequentemente nas sessões inicial e final; Solicitação de relato apresentou maior frequência na sessão intermediária; Solicitação de reflexão ocorreu mais nas sessões inicial e intermediária; e, Facilitação apresentou maior frequência na sessão final.

Nos primeiros encontros Informação e Solicitação de reflexão apareceram com altas frequências nas sessões iniciais. De acordo com Gil (2001), é comum num processo de coaching que o coach ajude o coachee refletir, pensar para que ele estabeleça seus objetivos. Para isso, o profissional deve dispor de informações precisas e fidedignas para auxiliar o cliente. Aparentemente é comum o coach dar explicações a respeito do coaching e esclarecer sobre procedimentos deste processo (Informação) nas sessões iniciais, assim como solicitar que o cliente reflita (Solicitação de reflexão) para estabelecer seus objetivos.

Solicitação de relato ocorreu com maior frequência na sessão intermediária. Zamignani (2007) aponta que Solicitação de relato ocorre ao longo de todo processo terapêutico, esta categoria é utilizada quando o terapeuta realiza coleta dados, solicita que o cliente faça descrições de comportamentos e quando o profissional solicita ao cliente informações sobre fatos. O que parece também ocorrer no coaching na relação coach-coachee. Apesar da categoria se apresentar frequente na terapia e no coaching é provável que a função em cada intervenção seja diferente, -na terapia o objetivo pode ser levantar informações para a elaboração da avaliação/interpretação (e.g., Leonardi, Borges \& Cassas, 2012), enquanto que no coaching a função de Solicitar informações pode ser para fazer com que o cliente observe seu comportamento para que ele próprio ganhe autoconhecimento e posteriormente possa estabelecer relações entre os eventos - pois não é papel do Coach levantar informações para avaliar ou interpretar.

Referente à categoria Facilitação, verifica-se que foi mais frequente na última sessão analisada. Hipotetiza-se que isso se deve pelo cliente já estar mais capacitado a se auto-observar, autoanalisar e autocontrolar, exigindo menos participação do coach, pois, de acordo com Borges (2015), estes comportamentos são ensinados no coaching.

Corrobora a hipótese de que a Facilitação está relacionada com maior autonomia do cliente o fato deste resultado coincidir com o aumento da frequência de relatos do cliente. Assim, é possível que o profissional ficasse mais exercendo função de facilitador dos relatos de auto-observações, autoanálises e autocontrole do cliente. Zamignani (2007) descreve que Facilitação indica atenção ao relato do cliente e sugere que ele continue falando. Esta categoria é caracterizada por pequenas verbalizações do terapeuta ou expressões paralinguísticas que ocorrem ao longo da fala do cliente.

A Figura 2 apresenta os resultados obtidos a partir da categorização dos comportamentos verbais emitidos pelo cliente durante as três reuniões de coaching analisadas. Foram encontradas semelhanças na frequência de parte das categorias das sessões categorizadas com os resultados de outros estudos cujo foco era a psicoterapia.

A partir dos dados apresentados na Figura 2, as categorias verbais vocais do cliente que ocorreram com maior frequência no processo de coaching foram Relato, Relação, Concordância e Solicitação. Já as respostas verbais vocais menos frequentes foram Melhora, Meta e Oposição. 


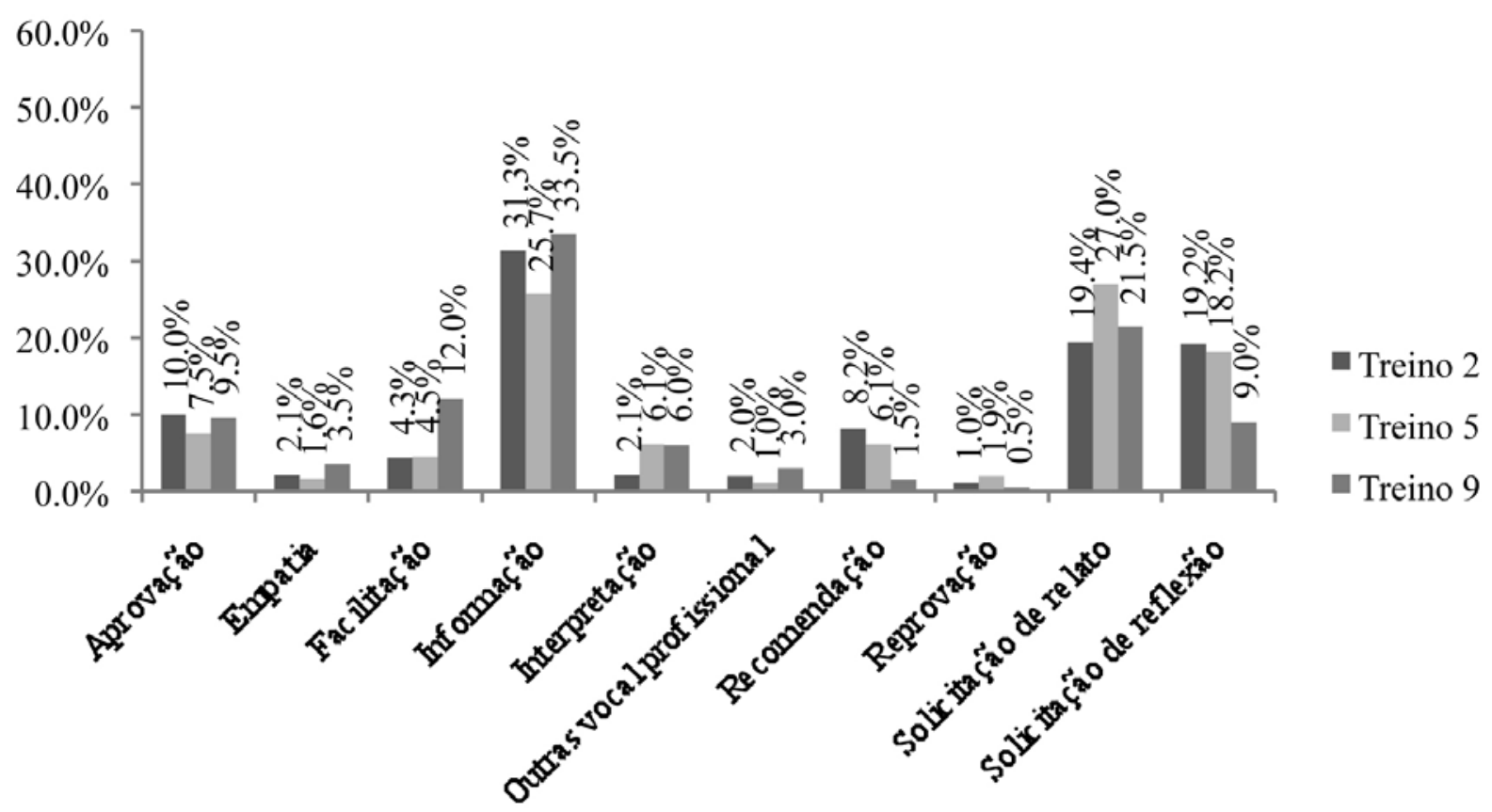

Figura 2: Percentual de ocorrência dos comportamentos verbais emitidos pelo cliente durante as sessões $n^{\circ}$ 02, 05 e 09.

Segundo Sadi e Meyer (2014), a condução do profissional influência nos comportamentos do cliente, ressaltando que a audiência não punitiva pode aumentar a probabilidade deste cliente confiar no profissional. As autoras acreditam que isso influencia na tendência do cliente emitir mais Relatos e ter baixa incidência da categoria Oposição.

Uma vez que a função do coach é evocar observações e reflexões em seu cliente por meio de técnicas utilizadas durante o processo (Gadelha \& Teodoro, 2014), nota-se que as categorias Reflexão e Relato do coachee aparecem como respostas às falas do profissional de coaching, quando este solicita ao cliente um relato ou reflexão de algo. Assim, observou-se, também, a relação entre a diminuição da categoria Solicitação de reflexão pelo coach, de modo que esta aparentemente interferiu na diminuição da categoria Relações do coachee ao longo da sessão intermediária e final.

As categorias com menor ocorrência de respostas verbais vocais foram Meta e Oposição, sendo esse resultado semelhante ao observado em estudos a respeito de interação terapêutica (e.g. Sadi \& Meyer, 2014; Zamignani \& Meyer, 2014), que tam- bém indicaram essas categorias ocorrendo com menor frequência.

É provável que a pouca frequência de ocorrências de Meta possa dever-se a essas já estarem estabelecidas na primeira sessão de coaching. A despeito disso, o dado chama atenção, visto que em processos de coaching, toda discussão é guiada pelos objetivos/metas.

Em relação à categoria Oposição é esperada sua baixa frequência, pois no processo de coaching espera-se que o profissional não puna os relatos do cliente, mas sim o apoie e o instigue a aceitar seu funcionamento e buscar seu desenvolvimento, o que pode diminuir o índice de Oposição e aumentar a ocorrência de relatos pelo coachee.

A categoria Melhora apresentou maior frequência ao final do processo de coaching, na última sessão categorizada (aumento de 10\% em relação à primeira sessão categorizada), podendo estar relacionado com o aspecto de que o processo já estava chegando ao seu final e o cliente notou avanços em seu comportamento em relação ao objetivo estabelecido, pois como defende Chiavenato (2005) e Borges (2015), o coaching é uma oportunidade para 
o indivíduo melhorar sua performance, melhorar comportamentos que já apresenta e/ou aprender os que carece.

Outro dado que se apresenta é o total de verbalizações do profissional e do cliente durante as reuniões de coaching. Tais resultados se encontram na Tabela 1, indicando o número de verbalizações de cada um em cada uma das sessões avaliadas.

Tabela 1: Ocorrência de verbalizações de cada sessão Coach-cliente

\begin{tabular}{|llll|}
\hline & Sessão 2 & Sessão 5 & Sessão 9 \\
\hline Coach & 498 & 358 & 194 \\
\hline Cliente & 498 & 357 & 197 \\
\hline
\end{tabular}

Pode-se dizer que o número absoluto de verbalizações em sessões de coaching foi maior que em sessões de psicoterapia (e.g. Oliveira, 2014). Contudo, a maior quantidade de verbalizações parece se dever ao tempo maior das sessões de coaching, isso porque, avaliando a quantidade relativa de verbalizações em relação ao tempo, encontram-se números semelhantes. No presente estudo o número de verbalizações por minuto variou entre 2,69 e 4,26, enquanto que no estudo de Oliveira (sobre terapia) variou entre 2,18 e 4,25.

Dada a semelhança na quantidade de verbalizações ocorridas nos dois processos, sugere-se que estudos futuros investigassem a duração dessas verbalizações, para verificar se elas também se assemelham.

\section{Considerações finais}

Apesar do crescimento do coaching nos últimos anos, há um déficit grande de estudos relacionados ao tema, especialmente no que se refere aos processos que ocorrem no processo de coaching. Nesse sentido, o presente estudo teve como propósito categorizar e analisar os comportamentos verbais vocais que ocorrem num processo de $c o$ aching, utilizando como base para categorização o Sistema Multidimensional para a Categorização de Comportamentos na Interação Terapêutica.

No processo de coaching, observou-se que os comportamentos do profissional mais frequentes foram Informar, Solicitar relatos e Solicitar reflexões, diferindo dos que são mais comuns em processos psicoterápicos - Interpretar e Empatizar. Esses dados sugerem que as práticas de coaching e psicoterapia são distintas.

Apesar de práticas diferentes, parece haver comportamentos em comum entre o terapeuta e o coa$c h$, pois os resultados indicam que no coaching, assim como na terapia, não é frequente o profissional discordar, reprovar e/ou se opor ao que o cliente diz.

Outro hipótese que pode se levantar a partir dos resultados é que no processo de coaching há maior quantidade de verbalizações que em processos terapêuticos. Contudo, essa diferença parece ser devido ao maior tempo das sessões de coaching e não a um maior número de verbalizações por minuto.

Por fim, é importante destacar também a eficácia de um sistema de observação assim como o SiMCCIT para a contínua evolução de pesquisas tanto relacionadas à terapia quanto ao coaching; sendo este sistema, capaz de produzir resultados descritivos de acordo com os comportamentos que ocorrem em sessão, bem como auxiliar na compreensão do funcionamento de um processo de coaching.

Vale lembrar que o presente estudo analisou um único processo de coaching sendo este um aspecto a se considerar, especialmente quando se pretende fazer generalizações a partir desses dados. Assim, sugere-se que mais estudos de processo sejam realizados a respeito de processo em coaching, seja utilizando-se desse mesmo método ou de outros, visando aumentar a compreensão a respeito da interação coach-coachee ou aumentar o poder de generalização destes resultados. Além disso, novos estudos podem contribuir, ainda, com as comparações entre os processos de coaching e psicoterapia, auxiliando na identificação de semelhanças e diferenças entre essas modalidades de intervenção. 


\section{Referências}

Ascama, M. J. O. (2004). Atuação do psicólogo e de outros profissionais no coaching em organizações de trabalho. Dissertação de Mestrado. Universidade Federal de Santa Catarina, Santa Catarina.

Banaco, R. A. (1993). O impacto do atendimento sobre a pessoa do terapeuta. Temas em Psicologia, 1(2), 71-79.

Banaco, E. A. (2012). Prefácio. Em: N. B. Borges \& F. A. Cassas (Orgs.), Clínica analítico-comportamental: aspectos teóricos e práticos (pp. ix-x) Porto Alegre: Artmed.

Blanco, V. B. (2006). Um estudo sobre a prática de coaching no ambiente organizacional e a possibilidade de sua aplicação como prática de gestão de conhecimento. Dissertação de Mestrado. Universidade Católica de Brasília, Brasília.

Block, V., Mendes, J., \& Visconte, L. (2012). Coaching executivo: uma questão de atitude. Rio de Janeiro: Elsevier.

Borges, N. B. (2015). Coaching AnalíticoComportamental: Estudos sobre efetividade de coaching feito por um analista do comportamento. Tese de Doutorado. Pontifícia Universidade Católica de São Paulo, São Paulo.

Braga, G. L. B., \& Vandenberghe, L. (2006). Abrangência e função da relação terapêutica na terapia comportamental. Estudos de Psicologia (Campinas), 23(3), 307-314.

Campos, T. M., \& Pinto, H. M. N. (2012). Coaching nas Organizações: uma revisão bibliográfica. REUNA, 17(2), 15-26.

Chiavenato, I. (2002). Construção de talentos: coaching \& mentoring. Rio de Janeiro: Elsevier.

Chiavenato, I. (2005). Construção de talentos: As novas ferramentas de gestão de pessoas. Rio de Janeiro: Elsevier.

Clutterbuck, D. (2008). Coaching Eficaz: Como orientar a sua equipe para potencializar resultados. São Paulo: Editora Gente.

Del Prette, G., Del Prette, Z. A., \& Meyer, S. B. (2007). Psicoterapia com crianças ou adultos: Expectativas e habilidades sociais de graduandos de psicologia. Estudos de Psicologia (Campinas), 24(3), 305-314.
Del Prette, G., \& Meyer, S. B. (2014). Categorização em terapia analítico-comportamental infantil: questões metodológicas e resultados de pesquisa. Em: D. R., Zamignani (Orgs.), A pesquisa de processo em psicoterapia: estudos a partir do Instrumento SiMCCIT (pp. 21-38). São Paulo: Paradigma.

Elisa, S. A. M., \& Yoshida, E. M. P. (2007). Coaching de executivos: adaptação e estágio de mudanças. Psicologia: teoria e prática, 9(1), 86-99.

Franchi, G., \& Sierra, P. A. (2007). Coaching: processos, técnicas e metodologias. Trabalho de Conclusão de Curso. Universidade São Judas Tadeu, São Paulo.

Gadelha, C. S., \& Teodoro, F. S. (2014). Coaching: ferramenta de desenvolvimento. Monografia. Faculdade de Pindamonhangaba, São Paulo.

Gil, A. (2001). Gestão de pessoas. São Paulo: Editora Atlas.

Grant, A. M. (2013). The Efficacy of Coaching. Em: J. Passmore, D. B. Peterson, \& T. Freire (Orgs.), The Wiley-Blackwell Handbook of the Psychology of Coaching and Mentoring (pp. 1539). Chichester, UK: John Wiley, \& Sons.

Guedes, M. L. (1993). Equívocos da terapia comportamental. Temas em Psicologia, 1(2), 81-85.

Guilhardi, H. J. (2004). Terapia por contingências de reforçamento. Em: C. N., Abreu \& H. J. Guilhardi (Orgs.), Terapia comportamental e cognitivo-comportamental: Práticas clínicas (pp. 3-40). São Paulo: Roca.

Karawejczyk, T. C., \& Cardoso, A. P. (2012). A atuação profissional em coaching e os desafios presentes e futuros nesta nova carreira. Boletim Técnico Senac, 38(1), 47-59.

Ireno, E. M. (2007). Formação de terapeutas analítico-comportamentais: efeitos de um instrumento para avaliação de desempenho. Dissertação mestrado. Universidade de São Paulo, São Paulo.

Leonardi, J. L., Borges, N. B., \& Cassas, F. A. (2012). Avaliação funcional como ferramenta norteadora da prática clínica. Em: N. B. Borges \& F. A. Cassas (Orgs.), Clínica AnalíticoComportamental: aspectos teóricos e práticos (pp. 105-109). Porto Alegre: Artmed.

Marçal, J. V. de S. (2010). Behaviorismo Radical e Prática Clínica. Em: A. K. C. R. de Farias (Org.), Análise comportamental clínica: aspectos teóri- 
cos e estudos de caso (pp. 30-48). Porto Alegre: Artmed.

Marques, J. R. (2013). Coaching \& Carreira: técnicas poderosas e resultados extraordinários: como o coaching pode transformá-lo em um profissional de sucesso. Goiânia: Editora IBC.

Meyer, S. B., \& Vermes, J. S. (2001). Relação terapêutica. Em: B. Range (Org.), Psicoterapias Cognitivo-Comportamentais (pp. 101-110). Porto Alegre, Artmed.

Moura, C. B., \& Venturelli, M. B. (2004). Direcionamentos para a condução do processo terapêutico comportamental com crianças. Revista Brasileira de Terapia Comportamental e Cognitiva, 6(1), 17-30.

Moskorz, L., \& Kubo, O. M. (2012). Desenvolvimento de um sistema multidimensional para a categorização de comportamentos na interação terapêutica: tese de doutorado de Denis Zamignani, 2007. Revista Brasileira de Terapia Comportamental e Cognitiva, 14(2), 64-69.

Oliveira, D. L., \& Borges, N. B. (2007). O ambiente natural como fonte de dados para a avaliação inicial e a avaliação de resultados: suplantando o relato verbal. Em: D. R. Zamignani, R. Kovac \& J. S. Vermes (Orgs.), A Clínica de Portas Abertas: experiências e fundamentação do acompanhamento terapêutico e da prática clínica em ambiente extraconsultório (pp. 77-100). São Paulo: Paradigma e Esetec.

Oliveira, E. M. (2009) Coaching e a abordagem clínica: alguns apontamentos epistemológicos. Toledo: Unioeste.

Oliveira, L. H. (2014). Analisando sessões de terapeutas comportamentais: dois estudos a partir da aplicação das categorias de Zamignani (2007). Em: D. R., Zamignani (Org.), A pesquisa de processo em psicoterapia: estudos a partir do Instrumento SiMCCIT (pp. 233-241). São Paulo: Paradigma.

Karawejczyk, T., \& Cardoso, A. (2012). Atuação profissional em coaching e os desafios presentes e futuros nesta nova carreira. Boletim Técnico SENAC, 38(1), 47-59.

Peron, F. (2012). Efeitos de uma atividade de fantasia em medidas da interação terapêutica. Dissertação de mestrado. Universidade Federal do Paraná, Paraná.
Sadi, H. M., \& Meyer, S. B. (2014). Análise da interação terapêutica em um caso de transtorno de personalidade borderline. Em: D. R. Zamignani (Org.), A pesquisa de processo em psicoterapia: Estudos a partir do Instrumento SiMCCIT (pp. 209-232). São Paulo: Paradigma.

Sant'Ana, V. L. (2004, outubro). A psicoterapia analítico-comportamental. Anais da Semana de Psicologia da Universidade Estadual de Maringá: Subjetividade e Arte, Maringá, PR, Brasil, 6.

Silva, C. R. E. (2010). Orientação Profissional, Mentoring, Coaching e Counseling: algumas singularidades e similaridades em práticas. Revista Brasileira de Orientação Profissional, 11(2), 299- 309.

Silveira, F. F. (2009). Análise da interação terapêutica em uma intervenção de grupo com cuidadoras. Dissertação de Mestrado. Universidade Estadual Paulista Júlio de Mesquita Filho, Bauru.

Simões Filho, E. F. (2014). Manejo de metáforas em psicoterapia analítico-comportamental. Qualificação de Mestrado. Universidade de São Paulo, São Paulo.

Starling, R. R. (2010). Prática controlada: medidas continuadas e produção de evidências empíricas em terapias analítico-comportamentais. Tese de Doutorado. Universidade de São Paulo, São Paulo.

Vandenbergue, L. (2011). Terceira onda e terapia analítico-comportamental: um casamento acertado ou companheiros de cama estranho?. Boletim Contexto, 34, 33-41

Vergara, S. O., Oliveira, S. Z., \& Ferreira, V. C. P. (2010). O papel do coach no processo de coaching, na percepção de coachees: um estudo com base na teoria das representações sociais. XXI Enangrad, Brasília.

Vernucio, R. R. (2012). Análise de sessões de terapia analítico-comportamental comparando sistemas de categorização. Iniciação científica. Universidade de São Paulo, São Paulo.

Vieira, A. L. C. (2013). Coaching: características do coach e benefícios do Coaching para o cliente. Dissertação de Mestrado, Instituto Politécnico do Porto, Pará.

Zamignani, D. R. (2007). O desenvolvimento de um sistema multidimensional para a categorização 
de comportamentos na interação terapêutica. Tese de Doutorado. Universidade de São Paulo, São Paulo.

Zamignani, D.R., \& Meyer, S. B. (2011) Comportamentos verbais do terapeuta no Sistema Multidimensional para a categorização de comportamentos na Interação Terapêutica (SiMCCIT). Revista Perspectivas, 2(1), 25-45.

Zamignani, D. R., \& Meyer, S. B. (2014). Aplicação do SiMCCIT em um conjunto de sessões de terapia Analítico-comportamental. Em: D. R. Zamignani (Orgs.), A pesquisa de processo em psicoterapia: o desenvolvimento do SiMCCIT (pp. 83-99). São Paulo: Paradigma.

Zamignani, D. R., \& Meyer, S. B. (2014). Apresentação. Em: D. R. Zamignani (Orgs.), A pesquisa de processo em psicoterapia: estudos a partir do Instrumento SiMCCIT (pp. 15-16). São Paulo: Paradigma. 